\section{HTH-14 A MULTI-CENTRE ANALYSIS OF AUGIB; UREA- CREATININE RATIOIS A USEFUL PREDICTOR FOR BLEEDING, AND ENDOTHERAPY}

\begin{abstract}
'Solange Serna, ${ }^{2}$ Jonathan Segal, ${ }^{3}$ Abdullah Abbasi, ${ }^{4}$ Junaid Aleem, ${ }^{4}$ Noor Alhamamy, ${ }^{3} \mathrm{Jad}$ Alkoury, ${ }^{5}$ Raheel Anjum, ${ }^{6} \mathrm{Mr}$ Benjamin Disney, ${ }^{3}$ Emma Howard, ${ }^{4}$ Aaron Hundle, ${ }^{4}$ Nasir Hussain, ${ }^{7}$ Mr Asem Ismail, ${ }^{8}$ Michael McFarlane, ${ }^{4}$ Ella Mozdiak, ${ }^{4}$ Saskia Port, ${ }^{9}$ Deepa Rattehalli, ${ }^{7}$ John Schembri, ${ }^{1}$ Geeth Silva, ${ }^{7}$ Mo Thoufeeq, ${ }^{1}$ Ajay Verma*. ${ }^{1}$ Kettering General Hospital NHS Foundation Trust; ${ }^{2}$ St Mary's Hospital, Imperial College Healthcare NHS trust; ${ }^{3}$ The Shrewsbury and Telford Hospital NHS Trust; ${ }^{4}$ Good Hope Hospital, University Hospitals Birmingham NHS Foundation Trust; ${ }^{5}$ The Royal Wolverhampton NHS Trust; ${ }^{6}$ University Hospitals Coventry and Warwickshire NHS Trust; ${ }^{7}$ Sheffield Teaching Hospitals NHS Foundation Trust; ${ }^{8}$ Warwick Hospital, South Warwickshire NHS Foundation Trust; ${ }^{9}$ The Dudley Group NHS Foundation Trust
\end{abstract}

10.1136/gutjnl-2021-BSG.65

Introduction The assessment of patients presenting with symptoms of acute upper GI bleed (AUGIB) is critical in determining if patients would benefit from a gastroscopy and endoscopically delivered therapy. The Rockall score, and superiorly, the Glasgow-Blatchford score (GBS) have been validated as effective risk tools for this purpose. The GBS considers serum urea levels, it established that digested blood from AUGIB causes a raised serum urea. This may be confounded by patients with kidney injury (acute, or acute on chronic) which may occur as a consequence of hypovolaemia in susceptible patients with AUGIB. There has been evidence that a urea creatinine ratio (UCR) may be useful identifying patients with a significant AUGIB. ${ }^{1}$ A preliminary analysis of 357 cases showed UCR - using a cut off of 97.7 was superior to serum urea (AUC 0.789 vs 0.733 ). ${ }^{2}$

Methods We collated data from 2203 presentations of AUGIB resulting in admission and an inpatient OGD within 72 hours, between January 2017 and January 2020, from 9 English centres. Data was recorded in a Microsoft Excel spreadsheet. We analysed serum urea levels, urea creatinine ratio (urea $\mathrm{x}$ 1000/creatinine), whether a plausible diathesis for AUGIB was seen, if endoscopic intervention was needed, and whether the patient had liver disease. Logistical regression was performed using SPSS.

Results Of the 2203 presentations (mean age 65.2 years, median 68, range 16-101 years), 1194 had a plausible bleeding diathesis (54.2\%), of this 1194, 721 had endoscopic therapy (60.4\%). 296 patients had liver disease (13.4\%) - of which 271 had a bleed (91.6\%).

Conclusions This large multicentre analyses of AUGIB cases have shown that UCR was a good indicator in AUGIB for patients who have a culprit lesion and required endoscopic therapy. It may be appropriate to use UCR as opposed to serum urea in patients with AUGIB when considering the need for endoscopic therapy.

\begin{tabular}{lllll} 
Abstract HTH-14 & Table 1 & & \\
\hline & All & $\begin{array}{l}\text { With liver } \\
\text { disease }\end{array}$ & $\begin{array}{l}\text { Without liver } \\
\text { disease }\end{array}$ & $\begin{array}{l}\text { Endoscopic } \\
\text { therapy }\end{array}$ \\
\hline Area under the curve & 0.7235 & 0.7409 & 0.7031 & 0.664 \\
$\begin{array}{l}\text { (AUC) } \\
\text { Threshold value }\end{array}$ & 101.6 & 86.1 & 101.6 & 101.2 \\
$\begin{array}{l}\text { Specificity } \\
\text { Sensitivity }\end{array}$ & 0.65 & 0.76 & 0.64 & 0.54 \\
\hline
\end{tabular}

\section{REFERENCES}

1. Srygley FD, Gerardo Cl, Tran T, Fisher DA. Does this patient have a severe upper gastrointestinal bleed? JAMA. 2012 Mar 14;307(10):1072-9.

2. Kotecha D, Mak J, Sharma N, et al. PTU-072|A multi-centre review of acute upper GI bleeding; can blood urea levels aid diagnosis? Gut 2019;68:A153.

\section{Small bowel}

\section{HTH-1 OPTIMUM STRATEGIES FOR IDENTIFYING ADULTS AND CHILDREN WITH COELIAC DISEASE (CD): AN EVIDENCE SYNTHESIS APPROACH}

${ }^{1}$ Hazel Everitt*, ${ }^{2}$ Athena Sheppard, ${ }^{2}$ Martha Elwenspoek, ${ }^{2}$ Howard Thom, ${ }^{2}$ Penny Whiting ${ }^{1}$ University Of Southampton, Southampton, UK; ' University of Bristol, Bristol, UK

\subsection{6/gutjnl-2021-BSG.66}

Introduction Guidelines recommend that adults and children 'at high risk' of CD should be offered testing. However, it is unclear which groups are at sufficiently high risk, which symptoms should prompt testing, and which tests should be offered. This NIHR funded study had five components: Systematic reviews on accuracy of tests for $\mathrm{CD}$; and on the predictive ability of diagnostic indicators for CD; Routine data analysis to develop prediction models; Collaborative work with patients to establish diagnostic thresholds; Cost-effectiveness analysis.

Methods Presented here are 2 systematic reviews using rigorous Cochrane methods on 1) the accuracy of tests for CD in adults and children and 2) the predictive ability of potential risk factors and symptoms for CD. Six databases were searched. Summary sensitivity, specificity and positive predictive values were estimated for each test and diagnostic indicator by fitting bivariate random effects meta-analyses.

Results 113 Studies evaluating serological test accuracy for CD diagnosis were included. Summary estimates for adults: IgA tTG (5 studies, 4310 participants, threshold $15 \mathrm{U} / \mathrm{ml}$ ) Sensitivity 90.7 (95\%CI, 87.3, 93.2) Specificity 87.4 (84.4, 90.0), IgA EMA (5 studies, 927 participants threshold 1:5) Sensitivity 88.0 (75.2, 94.7) Specificity 99.6 (92.3, 100.0). Summary estimates for children: IgA tTG (6 studies 2232 participants, threshold 20U/ml) Sensitivity 97.7 (91.0, 99.4) Specificity 70.2 (39.3, 89.6). IgA EMA (5 studies, 1257 participants threshold 1:10) Sensitivity $94.5 \quad(88.9,97.3) \quad$ Specificity $93.8 \quad$ (85.2, 97.5).

185 studies reporting 25 distinct diagnostic indicators were included. Analysis found large variation in diagnostic accuracy estimates between studies and high risk of bias. People with dermatitis herpetiformis, HLA DQ2/8 risk genotype, migraine, anaemia, type 1 diabetes, and osteoporosis were at least twice as likely as the general population to have CD. Gastrointestinal symptoms, psoriasis, epilepsy, inflammatory bowel disease, chronic liver diseases, systemic lupus erythematosus, fractures, type 2 diabetes, and multiple sclerosis showed poor diagnostic ability. People with first-degree relatives with CD had a three times higher risk of CD.

Conclusions Sensitivity and specificity estimates for test accuracy were lower than previously reported. IgA EMA specificity in adults could support serological diagnosis of CD. People with dermatitis herpetiformis, first-degree relatives, HLA DQ2/ 8 risk genotype, migraine, anaemia, type 1 diabetes, and osteoporosis have a higher risk of having $\mathrm{CD}$ than the general 